Service social

Comprendre pour soigner autrement, par Ellen E. Corin, Gilles

Bibeau, Jean-Claude Martin et Robert Laplante, Montréal, Les

Presses de l'Université de Montréal, 1990, 258 pages.

\title{
Diane Champagne
}

Volume 40, numéro 1, 1991

Éthique et intervention sociale

URI : https://id.erudit.org/iderudit/706522ar

DOI : https://doi.org/10.7202/706522ar

Aller au sommaire du numéro

Éditeur(s)

École de service social de l'Université Laval

ISSN

1708-1734 (numérique)

Découvrir la revue

Citer ce compte rendu

Champagne, D. (1991). Compte rendu de [Comprendre pour soigner autrement, par Ellen E. Corin, Gilles Bibeau, Jean-Claude Martin et Robert Laplante, Montréal, Les Presses de l’Université de Montréal, 1990, 258 pages.] Service social, 40(1), 148-150. https://doi.org/10.7202/706522ar d'utilisation que vous pouvez consulter en ligne.

https://apropos.erudit.org/fr/usagers/politique-dutilisation/ 
serait souhaitable de faire une place plus importante à l'analyse des comportements, des cognitions et de l'environnement social.

Le livre de Jean-Luc Hétu s'adresse principalement à des personnes aidantes semi-professionnelles (voir l'avant-propos). Il présente un modèle de type rogérien qui, dans l'état actuel de nos connaissances, ne peut prétendre représenter la seule façon ni même la meilleure façon de conduire une relation d'aide. À la décharge de l'auteur, remarquons que celui-ci discute d'une façon intéressante et souple de quelques méthodes d'intervention d'autres approches (psychanalytique, rationnelle-émotive, etc.). Mais, il s'agit généralement d'approches qui sont aussi centrées sur les émotions plutôt que sur les comportements ou sur l'environnement social. Quoi qu'il en soit, l'apport le plus original de ce livre réside sans doute dans un certain nombre de réflexions importantes, basées sur l'expérience clinique de l'auteur. Notons enfin que l'écriture de Jean-Luc Hétu est claire et précise et que son manuel est bien structuré et facile à consulter.

Jean-Marie Boisvert

École de psychologie

Université Laval

\section{Comprendre pour soigner autrement, par Ellen E. CORIN, Gil- les BIBEAU, Jean-Claude MARTIN et Robert LAPLANTE, Mont- réal, Les Presses de l'Université de Montréal, 1990, 258 pages.}

Dans cet ouvrage les auteurs présentent les résultats d'un travail de recherche conduit sur la question de l'adaptation des services de santé mentale à des réalités locales et régionales. L'approche qu'ils proposent s'inscrit dans un cadre conceptuel et une méthode anthropologique, qui vise à reconstruire le champ des perceptions et des pratiques en santé mentale en prenant en considération le point de vue des communautés. L'hypothèse qui est à la base de leur démarche est " que l'ensemble des perceptions et des représentations relatifs aux problèmes de santé se reflètent directement dans les réactions et dans les actions qu'ils suscitent et influencent notamment la place accordée aux ressources professionnelles» (p. 19).

L'originalité de l'ouvrage est de donner un modèle intéressant de ce qui pourrait être une véritable régionalisation des services de santé en proposant une approche socioculturelle des problèmes de santé mentale. C'est à l'application de cette méthode que nous invitent les auteurs à travers l'étude des problèmes de santé mentale dans trois milieux de l'Abitibi (forestier, agroforestier et minier) qui se sont développés à des périodes historiques différentes.

L'ouvrage comprend six chapitres qui nous entraînent à travers un recadrage anthropologique vers une compréhension des dynamiques locales et des problèmes de santé mentale dans la région de l'Abitibi.

Le premier chapitre nous situe par rapport aux grandes orientations des différents projets de réforme des services québécois de santé, pour ensuite dans le second chapitre nous présenter le contexte social et culturel de la région de l'Abitibi organisé autour de trois grands secteurs économiques primaires : minier, forestier et agro-forestier. Six villes et villages ont été retenus 
pour l'étude en tenant compte de l'ancienneté de l'axe de peuplement et de l'économie qui prédomina. Le travail d'analyse a été conduit du point de vue monographique afin de cerner les dynamiques communautaires propres à chacun des six milieux. Pour sa part le chapitre trois nous rappelle les données épidémiologiques et statistiques relatives aux problèmes de santé mentale et à I'utilisation des services de psychiatrie en Abitibi.

Le chapitre quatre constitue le "cœur " de l'ouvrage en ce sens que le travail d'analyse fait ressortir ce que les auteurs nomment "les nœuds" autour desquels s'organisent les systèmes de signes, de sens et d'actions dans chacune des localités ainsi que leur ancrage respectif dans les sous-cultures locales (p. 23).

Les récits les plus souvent évoqués dans les localités sont, par ordre d'importance, les cas de suicides ou de tentatives de suicides, les comportements violents associés à l'alcool, les problèmes de dépression, la négligence à s'habiller et à se nourrir et les personnes souffrant des conséquences d'un accident. Il est à propos de retenir de l'analyse des auteurs que les récits diffèrent selon la coloration des dynamiques des communautés à l'étude.

Le chapitre cinq s'inscrit en continuité avec le chapitre précédent en nous offrant une lecture anthropologique des problèmes de santé mentale en Abitibi, en dégageant les lignes de force de l'ensemble de la région et des particularités des localités. En outre, les auteurs relèvent un sentiment de précarité qui transparaît dans les six localités à l'étude et qui les amène à mettre en place différentes stratégies de protection. Cette lecture sensibilise les intervenants à l'importance de tenir compte de la signification populaire des problèmes de santé mentale si l'on veut rejoindre véritablement les populations.

Le sixième et dernier chapitre analyse les philosophies d'intervention des services abitibiens de santé mentale et dégage quatre grands modèles de pratique. Le premier se structure autour du travail infirmier, le second s'identifie aux approches psychologiques, le troisième est représenté par un modèle médico-centrique et le dernier, en récente émergence, est celui du modèle centré sur l'espace transitionnel situé entre les institutions et la vie quotidienne.

Les auteurs posent avec acuité l'impact de l'arrivée de nouveaux acteurs (psychiatres permanents, nouveaux CLSC, nouvelle politique de santé mentale) venant influencer la culture organisationnelle des services de santé mentale en Abitibi. Ce chapitre se conclut par l'analyse du point de vue des intervenants concernant la façon de voir les problèmes de santé mentale en Abitibi. Il s'agit par ordre d'importance d'une lecture médico-épidémiologique, géo-écologique, socio-économique, clinico-psychologique et culturelle.

En conclusion, les auteurs plaident en faveur d'un changement de paradigme et invitent planificateurs et professionnels de la santé mentale à travailler à l'articulation entre les dynamiques individuelles et collectives, contrairement au modèle dominant qui est peu soucieux de l'adéquation des services aux caractéristiques sociales et culturelles des communautés locales.

L'utilisation de la méthode anthropologique est très intéressante pour favoriser une compréhension des perceptions et des pratiques en santé mentale du point de vue des communautés et ainsi développer des services qui rejoignent véritablement les communautés. Le vocabulaire " anthropologique " utilisé peut toutefois être un peu difficile d'accès pour des non-initiés. Par ailleurs, la démarche des auteurs est intéressante et stimulante.

Une question qui demeure est à savoir si les six communautés à l'étude sont représentatives de la région de l'Abitibi, ou si on ne pourrait pas 
les comparer avantageusement à d'autres communautés minières, forestières, agro-forestières d'autres régions ayant connu la même trajectoire de peuplement, dépeuplement ? Concernant le chapitre sur l'analyse des philosophies des intervenants, il aurait été intéressant que cette culture organisationnelle soit plus exhaustive. Cet ouvrage devrait susciter un grand intérêt chez les professionnels et les administrateurs de différents champs de pratique par sa lecture socioculturelle des problèmes et son questionnement critique des pratiques.

Si l'ouvrage s'est voulu théorique et pratique, il peut laisser en appétit sur l'articulation d'un tel modèle dans la pratique de l'intervention. Dans la conclusion les auteurs avancent quelques paramètres sur le plan de l'action.

En résumé, il s'agit d'un excellent ouvrage qui incite à recadrer l'intervention plus près des particularités des communautés locales et à éviter le piège de la standardisation des services.

Diane Champagne

Université du Québec Rouyn-Noranda 